\title{
Mapeamento de áreas de subsidência com GPR em Teresina, PI
}

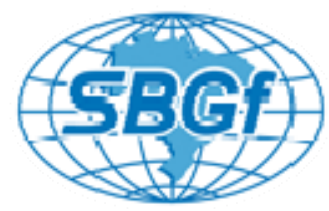

Alexandre Lisboa Lago (DISEGE/ERJ, CPRM), Welitom Rodrigues Borges (Instituto de Geociências, UnB), Elizângela Soares Amaral (RETE, CPRM)

Copyright 2019, SBGf - Sociedade Brasileira de Geofísica

This paper was prepared for presentation during the $16^{\text {th }}$ International Congress of the Brazilian Geophysical Society held in Rio de Janeiro, Brazil, 19-22 August 2019.

Contents of this paper were reviewed by the Technical Committee of the $16^{\text {th }}$ International Congress of the Brazilian Geophysical Society and do not necessarily represent any position of the SBGf, its officers or members. Electronic reproduction or storage of any part of this paper for commercial purposes without the written consent of the Brazilian Geophysical Society is prohibited.

\section{Abstract}

In order to identify possible shallow caves and associated structures, the Brazilian Geological Service (CPRM) developed a GPR campaign to verify the occurrence of reflection patterns characteristic of these dissolution structures. In the field, the team conducted GPR sections along streets with a history of collapse. The results of GPR obtained with the shielded antennas of $200 \mathrm{MHz}$ allowed the identification of old areas of collapse of the terrain, nevertheless until the maximum depth of 3 meters. The high content of clay present in the subsoil does not allow the GPR to investigate further depths in the research area, which eliminates its use for this purpose.

\section{Introdução}

A cidade de Teresina-PI apresenta um histórico de processos de afundamento de terreno. Dois eventos de afundamento de terreno merecem destaque, o primeiro, ocorrido em 1999 na Rua Simplício Mendes (Figura 1) e o outro ocorrido em 31 de julho de 2008, na Rua Francisco Mendes.

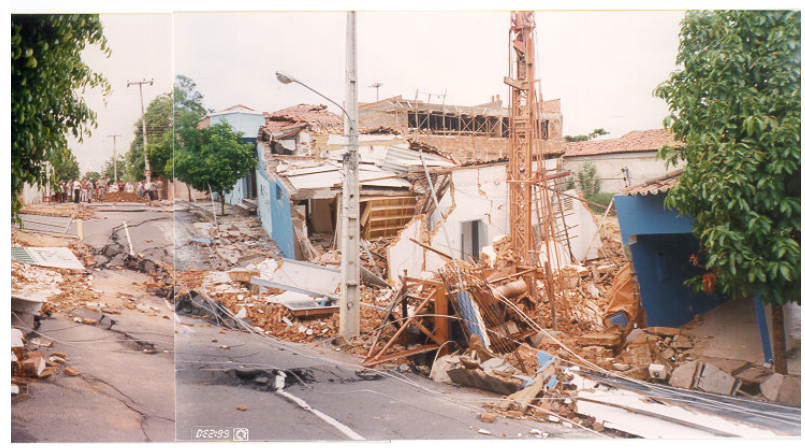

Figura 1 - Foto evidencia o colapso do terreno causado pela perfuração percursiva na rua Simplicio Mendes, Teresina, PI, no ano de 1999.

Ressalta-se, que a cidade de Teresina-PI vive atualmente um processo de acelerada verticalização dos espaços urbanos, com a construção de prédios altos e de elevado padrão. Entretanto, não é possível afirmar se, as características geotécnicas dos solos e rochas de
Teresina e as suas fragilidades, são de amplo conhecimento do meio técnico e principalmente das construtoras que atuam no município.

Face ao exposto, a natureza não invasiva dos métodos geofísicos, aliados ao baixo custo operacional e rapidez e facilidade de realização do levantamento, torna-os uma excelente ferramenta para ser aplicada no imageamento de áreas suscetíveis a afundamento de terreno na cidade de Teresina-PI. Além disso, algumas técnicas de levantamentos geofísicos fornecem dados contínuos sobre o local investigado, reduzindo muito a necessidade de dados diretos e específicos, assim como permitem uma análise temporal e espacial mais detalhada.

\section{Geologia}

A cidade de Teresina desenvolveu-se principalmente sobre rochas sedimentares incluídas na Formação Pedra de Fogo (Permiano), que consiste de horizontes alternados de arenitos médios a finos com estratificação cruzada e horizontes descontínuos de silexito e horizontes carbonáticos. $\mathrm{Na}$ base da formação Pedra de Fogo, ocorrem os arenitos brandos, cauliníticos finos que são muito friáveis. Essas litologias ocorrem sobrepostas aos sedimentos da Formação Piauí.

Os sedimentos da Formação Pedra de Fogo são suscetíveis a processos de dissolução que ocorre nos níveis carbonáticos e que podem estar condicionando os processos de colapso que ocorrem de forma ampla, na região central da cidade de Teresina.

\section{Metodologia}

O GPR é um método eletromagnético que emprega ondas de rádio em altas frequências (normalmente entre $10 \mathrm{MHz}$ a $2500 \mathrm{MHz}$ ) para identificar estruturas e feições geológicas rasas de subsuperfície ou localizar objetos enterrados pelo homem (PORSANI, 1999). O princípio físico e a metodologia de aquisição de dados GPR são semelhantes à técnica de reflexão sísmica e a técnica de sonar, com exceção de que o GPR é baseado na reflexão de ondas eletromagnéticas (ANNAN, 1992).

O método GPR é comumente usado em estudos geotécnicos que envolvam a identificação de cavidades no subsolo em função da agilidade de obtenção dos registros e dos resultados (ANCHUELA et al, 2009; ANCHUELA et al, 2014).

Neste trabalho, para calibrar a aquisição de dados de GPR na área metropolitana de Teresina, realizaram-se investigações $2 \mathrm{D}$ em áreas com histórico de colapso de cavernas. 
Os dois eventos descritos neste trabalho referem-se a afundamentos de terreno provocados pela perfuração de cavernas, e consequentemente colapso das mesmas. Durante o campo usou-se o GPR acoplado a antenas blindadas de $200 \mathrm{MHz}$ (Figura 2). Neste trabalho apresentam-se aquisições de GPR realizadas na rua Francisco Mendes e na rua Simplício Mendes (Figura 3).
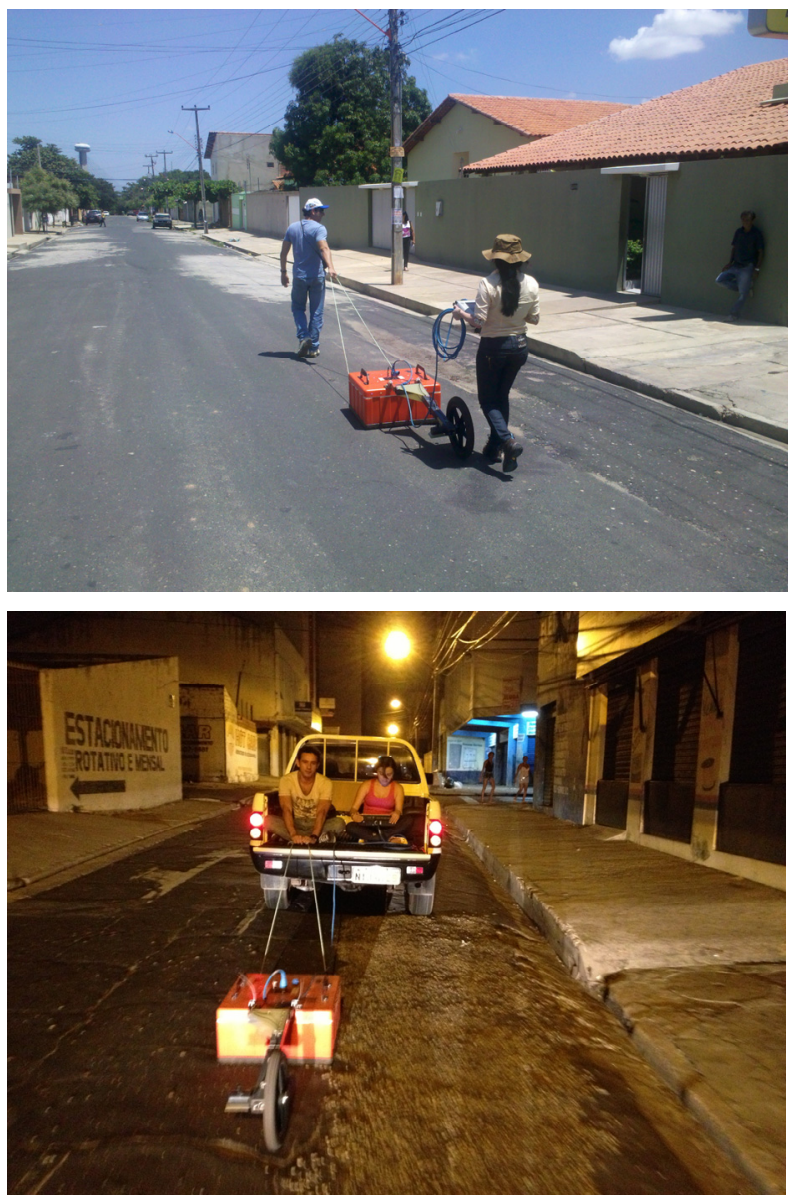

Figura 2 - Fotos evidenciam a aquisição de dados de GPR nas ruas de Teresina, PI.

Os dados foram adquiridos deslocando-se o GPR através da técnica do afastamento constante (common offset). Os parâmetros de aquisição de dados foram:

- As frequências de amostragem temporal foram de $1700 \mathrm{MHz}$ e $3400 \mathrm{MHz}$, resultando em janelas temporais de 300 ns e 150 ns, respectivamente;

- Para as janelas de 300 ns e 150 ns usou-se 1024 amostras por traço, propiciando assim, um intervalo de amostragem temporal de 0,2929 ns e 0,1464 ns respectivamente;

- O intervalo de amostragem entre os traços foi de $2,5 \mathrm{~cm}$ e $5 \mathrm{~cm}$. Esta amostragem foi realizada automaticamente com o uso de um odômetro eletrônico (roda).
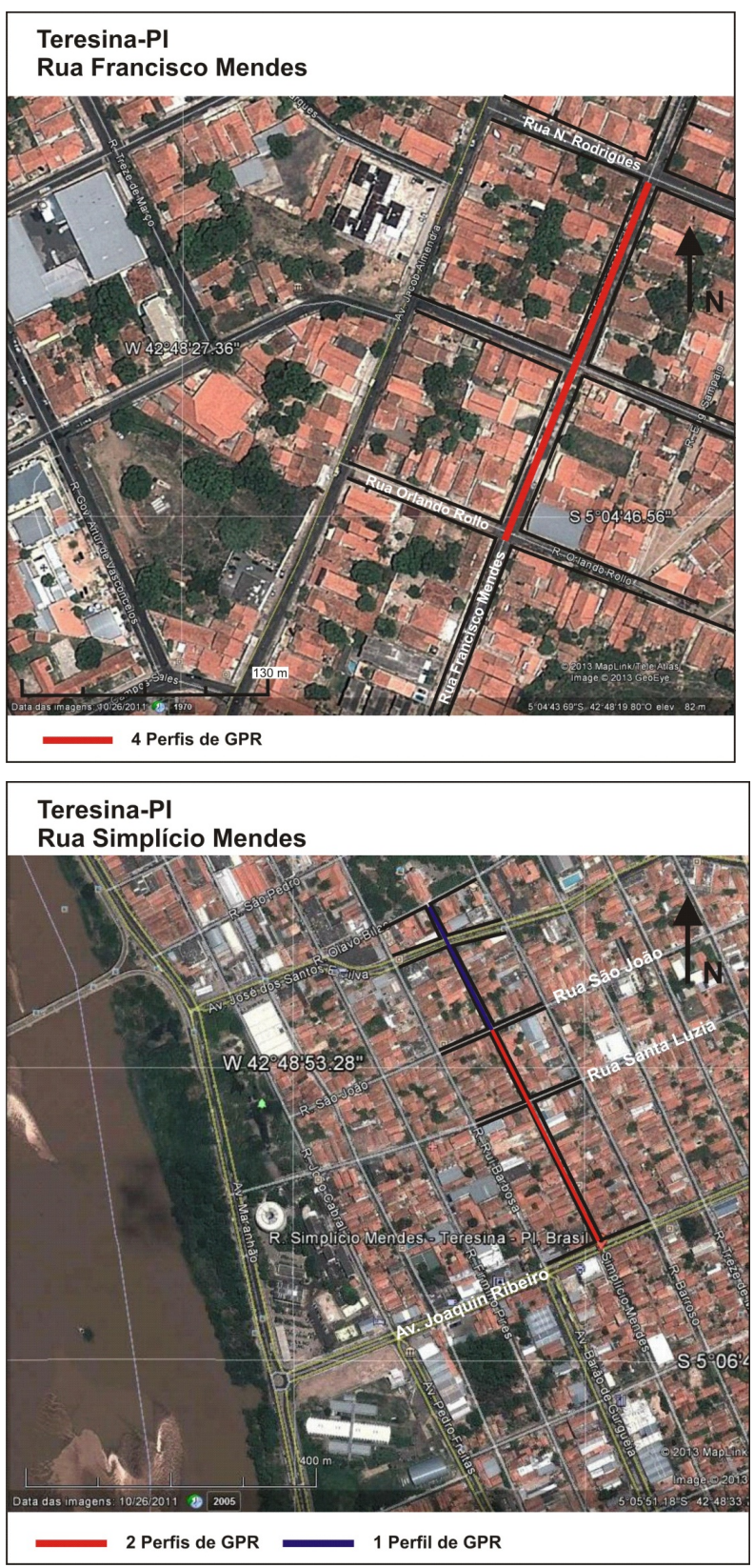

Figura 3 - Imagens aéreas da cidade de Teresina, com a localização das seções de GPR realizadas nas ruas Francisco Mendes e Simplício Mendes.

\section{Processamento}

O processamento dos dados de campo adquiridos pelo método GPR 2D foram processados no software ReflexW, versão 7.0 (SANDMEIER, 2012). A rotina de processamento dos dados 2D consistiu em:

- $\quad$ IMPORT - conversão de formato dos arquivos ( ${ }^{*}$.dzt - saída do equipamento SIR3000, para o formato *.dat - arquivo do ReflexW); 
- SET TIME ZERO (ajuste do tempo zero) ajuste das primeiras chegadas da onda eletromagnética;

- ENERGY DECAY (ganho) - aplicado para recuperação da amplitude do sinal eletromagnético atenuada durante a propagação do sinal no meio;

- BANDPASS (filtro 1D) - eliminação dos ruídos eletrônicos e estáticos inerentes ao sistema;

- BACKGROUND REMOVAL (filtro 2D) remoção de ruídos coerentes relacionados à reverberação da onda eletromagnética dentro da blindagem da antena e de ruídos externos;

- KIRCHHOFF MIGRATION - processo utilizado para colapsar as hipérboles de difração. A migração é um processo inverso no qual as ondas registradas são propagadas de volta à localização correspondente ao difrator;

- TIMEDEPTH CONVERSION - conversão dos perfis de tempo para profundidade. A velocidade de conversão, utilizada nesta etapa, foi obtida através do ajuste hiperbólico de alguns pontos difratores encontrados na área investigada.

A mesma rotina de processamento foi aplicada a todos os radargramas $2 \mathrm{D}$, com o intuito de comparação das amplitudes dos sinais. A velocidade média de propagação da onda eletromagnética, no solo, foi determinada pelo método do ajuste hiperbólico de pontos difratores relacionados a tubulações encontradas próximas à superfície (YILMAZ, 1987). O ajuste dos eventos hiperbólicos mostra que a média das velocidades adaptadas foi de $0,08 \mathrm{~m} / \mathrm{ns}$.

\section{Interpretação e Discussão dos Resultados}

A análise dos resultados de GPR é feita através da correlação entre a geometria e a amplitude dos eventos refletores. Nas seções de radar notam-se padrões distintos de reflexão que traduzem o comportamento elétrico do meio à passagem dos campos eletromagnéticos de altas frequências.

O primeiro padrão de reflexão observado (PR1) possui alta amplitude, refletores horizontalizados e inclinados, contínuos e descontínuos, e corresponde ao padrão característico de aterros e solos (Figura 4a). Este padrão é registrado em todas as seções na parte superficial das mesmas, ou seja, até a profundidade média de 2 metros.

O segundo padrão de reflexão (PR2) é caracterizado por refletores de baixa amplitude, caóticos e totalmente descontínuos (Figura 4b), e ocorre logo abaixo do PR1. Este padrão de reflexão marca a região em que o sinal eletromagnético do GPR é absorvido pelo meio. Esta absorção deve ser efeito da atenuação do sinal pela presença de camadas eletricamente condutivas, tais como argilitos, siltitos ou arenitos com matriz argilosa.

Além destes dois padrões de reflexão notam-se, nas seções de GPR, refletores hiperbólicos relacionados a interferências subterrâneas (tubulações, dutos, fios elétricos, etc., Figura 4c).
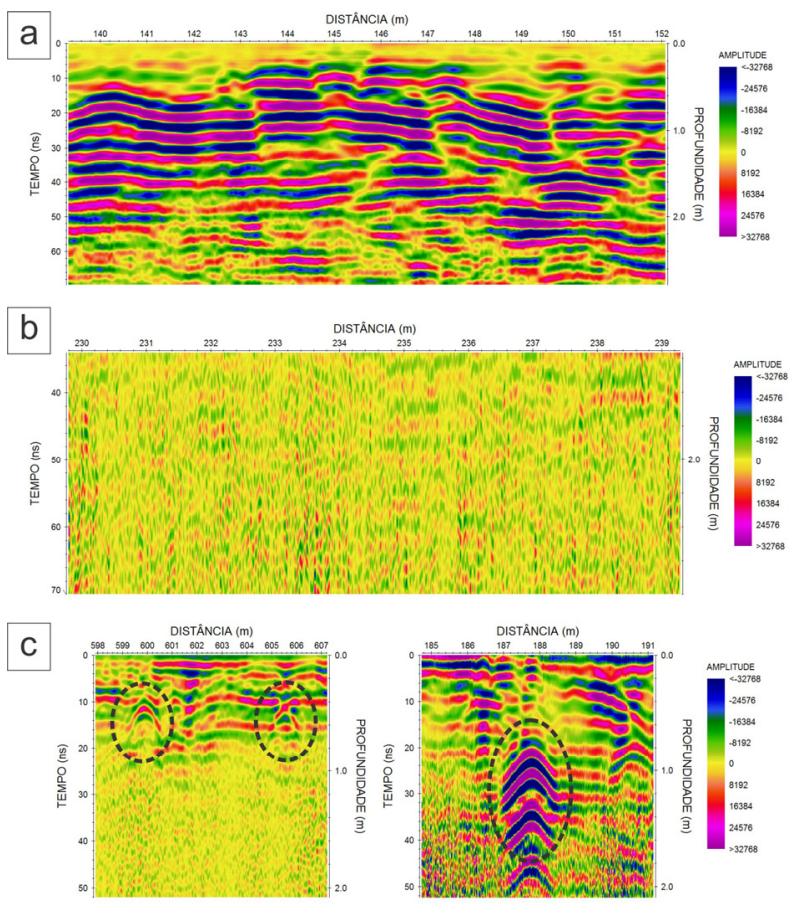

Figura 4 - Detalhes de seções de GPR realizadas na área urbana de Teresina/PI, onde se evidenciam os padrões de reflexão (PR1) relacionado aos solos e aterros (4a); ao padrão (PR2) relacionado à área de atenuação do sinal do GPR (zona condutiva, 4b); e os refletores hiperbólicos relacionados a interferências subterrâneas (canos, dutos, etc., 4c).

A seção de GPR realizada na Rua Simplício Mendes mostra uma deformação no padrão de reflexão PR1, resultado do afundamento do terreno ocorrido no dia 29 de dezembro de 1999 (Figura 5). Este afundamento ocorre na seção de GPR entre as posições 125 e 205 metros, com o centro na posição de 164 metros. Esta zona anômala é evidenciada pela inflexão de refletores.

A seção de GPR realizada na Rua Francisco Mendes mostra uma deformação no padrão de reflexão PR1, resultado do afundamento do terreno ocorrido no dia 31 de julho de 2008 (Figura 6). Este afundamento ocorre na seção de GPR entre as posições 36 e 67 metros, com o centro na posição de 57 metros. Esta zona anômala é evidenciada pela inflexão de refletores. 

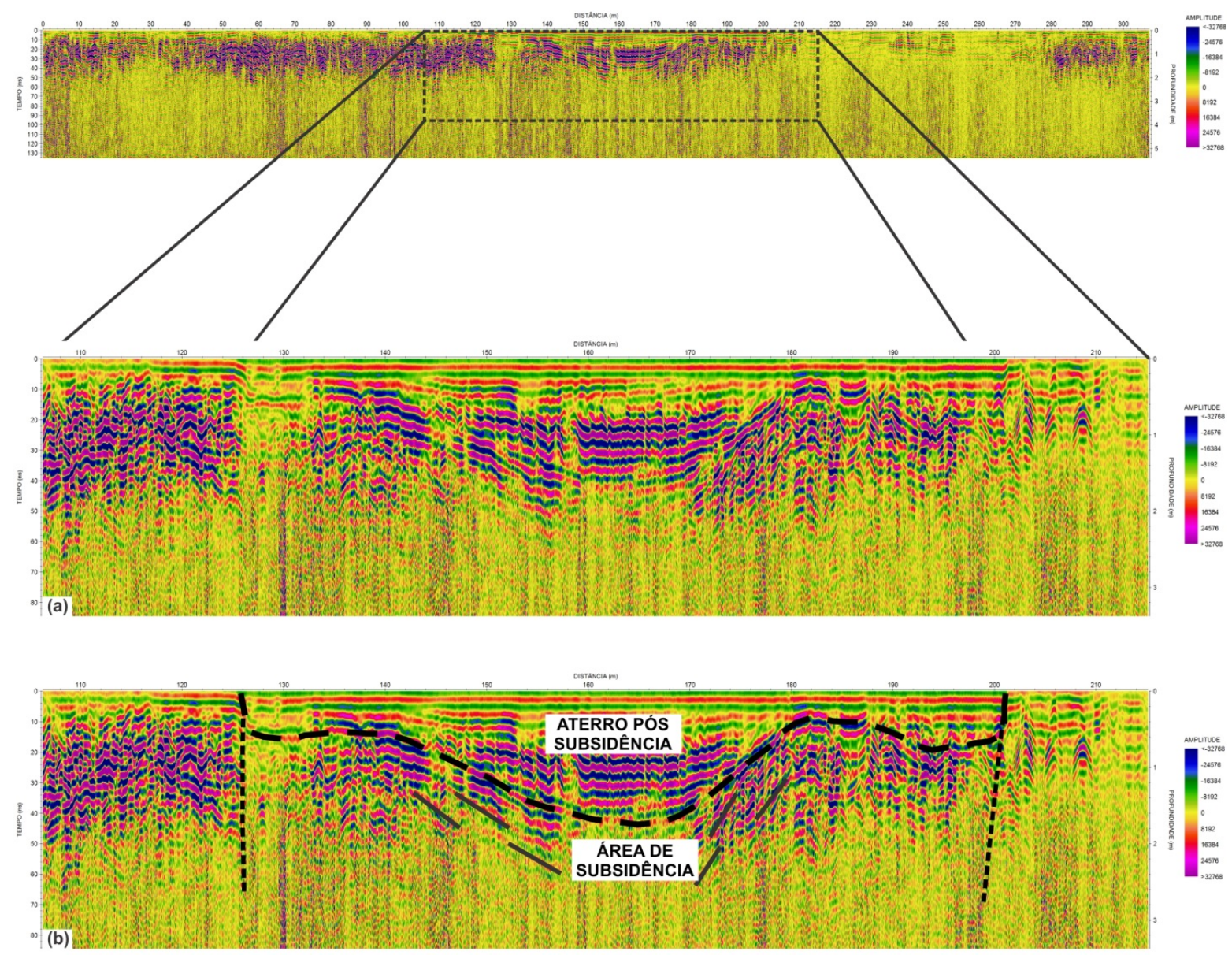

Figura 5 - Seção de GPR realizado na rua Simplício Mendes, Teresina/PI. (a) Detalhe da seção evidenciando o padrão de reflexão típico de áreas onde ocorreram subsidências. (b) Mesma seção interpretada.

\section{Conclusões}

As investigações de GPR realizadas nas ruas e avenidas de Teresina/PI evidenciam uma cobertura pedológica e/ou aterro até a profundidade máxima de 3 metros e alvos hiperbólicos relacionados a interferências subterrâneas (dutos, encanamentos, tubulações, etc.).

Nos locais onde há registro de subsidências (ruas Simplício Mendes e Francisco Mendes), os refletores de GPR sofrem um basculamento, ou seja, deixam de ser horizontalizados e inclinam-se na direção do centro da subsidência. Nas extremidades destas áreas nota-se também a interrupção destes refletores, sugerindo o rompimento das camadas geológicas. Este padrão é típico de solapamento de cavernas.

\section{Agradecimentos}

Os autores agradecem ao Serviço Geológico Brasileiro (CPRM) pelo financiamento do Projeto e ao Instituto de Geociências da Universidade de Brasília pela cessão do equipamento de GPR.

\section{Referências}

ANCHUELA, ÓP; CASAS-SAINZ, AM; SORIANO, MA; POCOVÍ-JUAN, A. 2009. Mapping subsurface karst features with GPR: results and limitations. Environ Geol, 58:391-399.

ANCHUELA, ÓP; CASAS-SAINZ, AM; POCOVÍ-JUAN, A; GARBÍ, HG; CALVÍN, P. 2014. Characterization of the Karstic Process in an Urban Environment Using GPR Surveys. J. Mater. Civ. Eng., 26(8): 05014004.

ANNAN, A.P. 1992. Ground penetration radar workshop notes. Sensor; Software, Inc., Internal Report, 130p.

PORSANI, J.L. 1999. Ground Penetrating Radar (GPR): Proposta metodológica de emprego em estudos geológico-geotécnicos nas regiões de Rio Claro e Descalvado-SP. Tese de Doutorado, Instituto de Geociências e Ciências Exatas, UNESP, Campus de Rio Claro-SP, 145p.

SANDMEIER, K.J. 2012. REFLEXW Version 7.0 for Windows $9 x / 2000 / N T / X P$. Program for the processing of seismic, acoustic or electromagnetic reflection, refraction and transmission data. Manual do Software, 192p. 

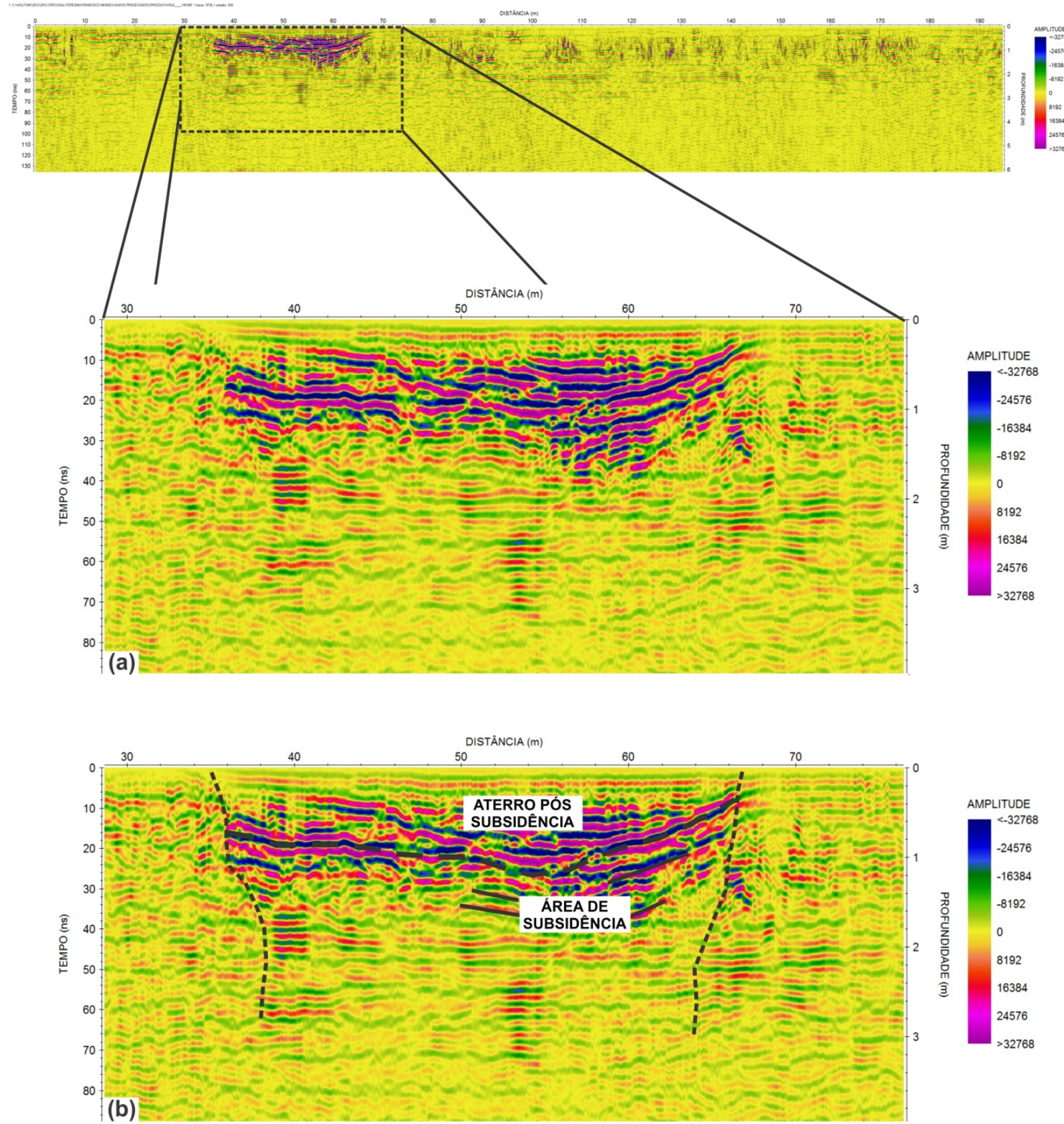

Figura 6 - Seção de GPR realizado na rua Francisco Mendes, Teresina/PI. (a) Detalhe da seção evidenciando o padrão de reflexão típico de áreas onde ocorreram subsidências. (b) Mesma seção interpretada. 